\title{
Interstellar hydrogen bonding
}

\author{
Emmanuel E. Etim ${ }^{\mathrm{a}, *}$, Prasanta Gorai ${ }^{\mathrm{b}}$, Ankan Das ${ }^{\mathrm{b}}$, Sandip K. Chakrabarti ${ }^{\mathrm{b}, \mathrm{c}}$, \\ Elangannan Arunan ${ }^{\mathrm{d}}$ \\ ${ }^{a}$ Department of Chemical Sciences, Federal University Wukari, Katsina-Ala Road, P.M.B. 1020 Wukari, Taraba State, Nigeria \\ ${ }^{\mathrm{b}}$ Indian Centre for Space Physics, 43 Chalantika, Garia Station Road, Kolkata 700 084, India \\ ${ }^{c}$ S. N. Bose National Centre for Basic Sciences, Salt Lake, Kolkata 700098, India \\ ${ }^{\mathrm{d}}$ Inorganic and Physical Chemistry Department, Indian Institute of Science Bangalore, 560012, India
}

Received 4 August 2017; received in revised form 3 February 2018; accepted 3 March 2018

Available online 14 March 2018

\begin{abstract}
This paper reports the first extensive study of the existence and effects of interstellar hydrogen bonding. The reactions that occur on the surface of the interstellar dust grains are the dominant processes by which interstellar molecules are formed. Water molecules constitute about $70 \%$ of the interstellar ice. These water molecules serve as the platform for hydrogen bonding. High level quantum chemical simulations for the hydrogen bond interaction between 20 interstellar molecules (known and possible) and water are carried out using different ab-intio methods. It is evident that if the formation of these species is mainly governed by the ice phase reactions, there is a direct correlation between the binding energies of these complexes and the gas phase abundances of these interstellar molecules. Interstellar hydrogen bonding may cause lower gas abundance of the complex organic molecules (COMs) at the low temperature. From these results, ketenes whose less stable isomers that are more strongly bonded to the surface of the interstellar dust grains have been observed are proposed as suitable candidates for astronomical observations.
\end{abstract}

(C) 2018 COSPAR. Published by Elsevier Ltd. All rights reserved.

Keywords: ISM; Abundance-ISM; Atoms-ISM; Molecules; Physical data and processes; Astrochemistry

\section{Introduction}

Gas phase chemical reactions and reactions that occur on the surfaces of interstellar dust particles are the dominant processes by which molecules can be synthesized from the precursor species. Of these two processes, reactions that occur on the surfaces of interstellar dust particles have been invoked for the formation of molecular hydrogen (Biham et al., 2001; Chakrabarti et al., 2006); as well as for the synthesis of larger interstellar molecules (Das et al., 2010; Das and Chakrabarti, 2011; Das et al., 2016). Dust grains and ice play an indisputable role for the synthesis of interstellar species. Whereas in the gas phase, reactions relying on

\footnotetext{
* Corresponding author.

E-mail address: emmaetim@gmail.com (E.E. Etim).
}

three-body collisions are very rare, species residing on the solid surfaces can roam around the surfaces until a reaction occurs, thus forming new species. These surfaces therefore serve as reaction sites for chemical processes that would either being very slow or not occur at all. From spectroscopic observations, the composition of the interstellar ice is dominated by water which accounts for $60-70 \%$ (Das and Chakrabarti, 2011; Das et al., 2016) of the ice in most lines of sight. Other spectroscopically observed species include methanol, carbon monoxide and carbon dioxide, as well as smaller abundances of other species (Tielens, 2013; Fraser et al., 2002; Draine, 2003; van Dishoeck, 2004; Gibb et al., 2000; Gibb et al., 2004; Whittet, 2003). The composition of the interstellar ice causes most of the molecules formed on the surface of the interstellar dust to form hydrogen bonding with the components of the ice most 
predominantly, water. This causes a greater part of these molecules to be attached to the surface of the interstellar dust grains, thus reducing its interstellar gas phase abundance.

Weak intermolecular interactions are very vital in the elucidation of structures and properties of many important biological molecules like water, DNA, protein etc. Among these weak intermolecular interactions, hydrogen bonding is well recognized, studied and understood to a great extent because of its overwhelming impacts in different systems and phenomena. It is responsible for the unique properties of water essential to life; it serves as a vital force in determining the basic structure of large bio-molecules such as DNA, RNA, proteins etc (Park and Lee, 2007). The binding energy of the hydrogen bonded complex gives information about the strength of the bonding. This binding energy can be determined both experimentally and theoretically. With the advances in quantum chemical calculations, on a careful choice of method (level of theory) and basis set, it is now possible to theoretically estimate binding energy that is in good agreement with the experimentally measured value.

As useful as the DFT method is, it has a number of drawbacks (especially for large systems); insufficient description of van der Waals interaction, inaccurate estimation of polarizabilities of large $\pi$-conjugate molecules. It has also been shown to underestimate the binding energy of complexes in comparison with the MP2 to MP4(SQD) methods. This underestimation is said to arise from the insufficient description of electron correlation effects. Among the available ab initio methods, the MøllerPlesset second order method (MP2) has been shown to be effective and accurate in investigating hydrogen-bonded systems; the use of the smallest augmented correlation consistent basis set (aug-cc-pVDZ) for hydrogen-bonded complexes is strongly recommended (Park and Lee, 2007; Ikeda et al., 2007; Møller and Plesset, 1943; Head-Gordon et al., 1988; Frisch et al., 1990).

The formation of interstellar species essentially depends on their formation pathways not on the thermodynamic parameters. However, some correlation between the observed molecules and thermodynamic parameters really exists wherever reaction pathway does not appear to influence. For example, recently, Etim et al. (2016) had shown that the detectability of the odd numbered carbon chains could be correlated due to the fact that they are more stable than the corresponding even numbered carbon chains. Some more studies by Etim et al. (2017) investigated the possibility of detecting most suitable isomer in the $\mathrm{C} 5 \mathrm{H} 9 \mathrm{~N}$ isomeric group. However, it is not true always. For example, Sil et al. (2017) investigated the possibility of detecting certain amines and aldimines, Gorai et al. (2017) investigated the possibility of detecting some complex thiols in the ISM based on various relevant parameters. They found that most stable species should not be considered as the most potential candidate always because interstellar chemistry is far away from the equilibrium. We also have noticed some deviations from the fact that most stable isomer is observed among some of the groups considered; the conspicuous abundance of a less stable isomer (methyl formate) over the most stable one (acetic acid) and the non-detection of some of the most stable isomers whose less stable counterparts have been detected.

In the present study, we discuss that if the formation/ destruction of some species are not influenced by their gas phase pathways and completely controlled by means of the grain surface reactions, gas phase abundances of these species will be strongly influenced by the binding energies. The binding energy of simple interstellar species like $\mathrm{H}_{2}, \mathrm{CO}, \mathrm{N}_{2}, \mathrm{CO}_{2}, \mathrm{CH}_{3} \mathrm{OH}, \mathrm{CH}_{4}, \mathrm{NH}_{3}, \mathrm{H}_{2} \mathrm{CO}$, etc. are well known but for the complex species, it is mostly unknown. Very recently, Wakelam et al. (2017) extensively studied the binding energy of simple as well as some complex interstellar species by using quantum chemical calculations. They considered a single water monomer as an adsorbent. Initially, they calculated the binding energy of 16 stable species and calibrated their values with the experimentally available BE. We have extracted their fitted values and found that if we use following relation then there values for the 16 stable species can be explained.

Scaled $\mathrm{BE}=\mathrm{A} 0+\mathrm{A} 1 \times$ Calculated $\mathrm{BE}$,

where A0 $=289.019$ and A1 $=1.65174$. From KIDA database it can be seen that uncertainties of their estimated values are mentioned to be $\sim 30 \%$ even after using the scaling factor. So, we think that after appropriate scaling our proposed $\mathrm{BE}$ values of the complex species would certainly help the community to build astrochemical model with an educated estimation rather than considering a blind guess.

We aim to account for these variations and try to correlate the difficulty in the astronomical observation of sugars and want to propose suitable candidates for astronomical observation within the range of our molecules under consideration. For the present study, 20 interstellar molecules (known and possible) which are suitable in achieving the aims of the present study stated above are considered. The strength of their hydrogen bonded complexes with water is investigated by estimating their binding energies at different levels of theory and basis sets. After describing the computational methods used here, the results obtained are presented and discussed.

\section{Computational details}

All the calculations reported in this work are performed using the Gaussian 09 suite of programs (Frisch et al., 2009). The geometry optimization of all the monomers, water and the corresponding hydrogen bonded complexes have been carried out using the Møller-Plesset second order perturbation theory; MP2(full) with the $6-311++G(d, p)$ and aug-cc-pVDZ basis sets, the G4 composite method and the Weizmann theory (W1U) (Møller and Plesset, 1943; Martin and de Oliveira, 1999; Parthiban and Martin, 2001; Curtiss et al., 1998; Curtiss et al., 2007a,b). 
The Gaussian G4 theory and the Weizmann theory are compound models that offer high accurate predictions at less computational cost. Diffuse functions in the basis sets allow the orbitals to occupy a larger region of space while the polarization functions give additional flexibility to the description of molecular orbitals (Chandrasekhar et al., 1981; Hariharan and Pople 1973). Harmonic vibrational frequencies were performed on all the systems (monomers, water molecule and complexes) to characterize their stationary nature with equilibrium structures having no imaginary frequency. The binding energy between water molecule and each interstellar molecule (known and possible) considered here is calculated as the energy of the complex minus the energies of the isolated monomers. It is defined by the following expression:

$\Delta \mathrm{E}_{(\text {binding energy })}=\mathrm{E}_{(\text {complex })}-\left[\mathrm{E}_{(\text {water molecule })}+\mathrm{E}_{(\text {interstellar molecule })}\right]$.

The reported binding energies have been corrected for the basis set superposition error (BSSE) using the counterpoise method developed by Boys and Bernardi (Boys and Bernardi, 1970).

\section{Result and discussion}

Binding energies for 20 hydrogen bonded complexes with water have been estimated at different levels of theory and basis sets. The full geometry parameters for water and all the complexes studied in this work are reported in the supporting information. Based on Wakelam et al. (2017), here, we scaled down our calculated values by the same factor to propose the binding energy values of the complex species considered in this study. We just extracted the data of Fig. 1 of Wakelam 2017 paper to find out the fitting coefficients using the fitting formula $\mathrm{Y}=\mathrm{A} 0+\mathrm{A} 1{ }^{*} \mathrm{X}$ and we get the value of coefficients, $\mathrm{A} 0=289.019$ and $\mathrm{A} 1=1.65174$. The results presented here are from the most stable structures of all the systems considered. After a brief description of the geometry parameters for water which is the central molecule for this study, the discussion will be presented in the subsections in accordance with the aims of this work.

The theoretically calculated geometry parameters of the water monomer at the different levels of theory and basis sets are listed in Table 1. The corresponding experimental

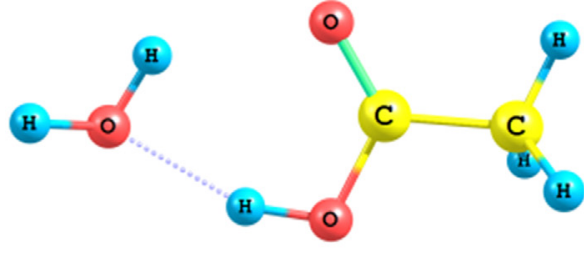

Acetic acid-water complex

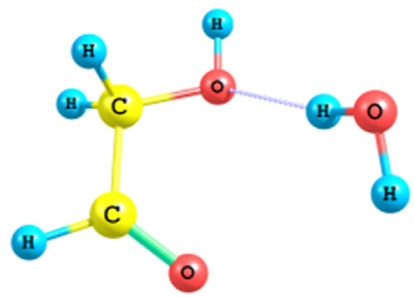

Glycolaldehyde-water complex

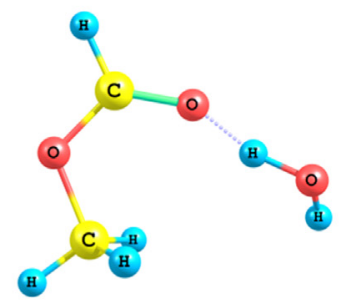

Methyl formate-water complex

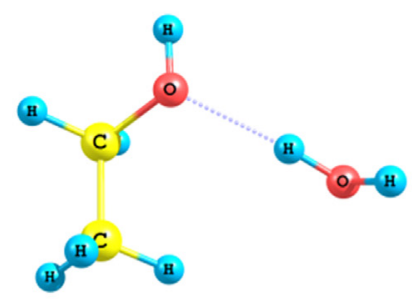

Ethanol-water complex

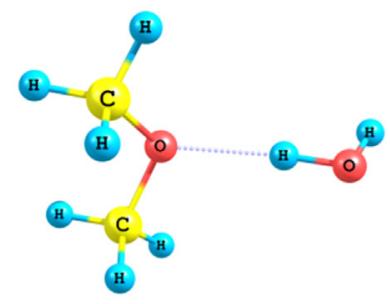

Dimethylether water complex

Fig. 1. Optimized structures of the hydrogen bonded $\mathrm{C}_{2} \mathrm{H}_{4} \mathrm{O}_{2}$ and $\mathrm{C}_{2} \mathrm{H}_{6} \mathrm{O}$ isomer complexes with water.

Table 1

Optimized geometry $(\AA$, deg) parameters of the water monomer.

\begin{tabular}{llllll}
\hline Parameters & Method & & & \\
\cline { 2 - 6 } & MP2(full)6-311++G & MP2(full)/aug-cc-Pvdz & G4 & W1U & Exptl \\
\hline $\mathrm{H}_{2}-\mathrm{O}_{1}$ & 0.959 & 0.966 & 0.962 & 0.961 & $0.959^{\mathrm{a}}$ \\
$\mathrm{H}_{3}-\mathrm{O}_{1}$ & 0.959 & 0.966 & 0.962 & 0.961 & $0.959^{\mathrm{a}}$ \\
$\mathrm{H}_{3}-\mathrm{O}_{1}-\mathrm{H}_{2}$ & 103.5 & 103.9 & 103.7 & 104.5 & $103.9^{\mathrm{a}}\left(104.5^{\mathrm{b}, \mathrm{c}}\right)$ \\
\hline
\end{tabular}

a 23 .

b 24.

c 25 . 
values from literature are included in the table (as exptl). The $\mathrm{OH}$ bond length predicted by the $\mathrm{MP}(\mathrm{full}) / 6-311+$ $+\mathrm{G}(\mathrm{d}, \mathrm{p})$ is exactly as the experimentally measured value. The $\mathrm{OH}$ bond length estimated by other methods employed here are in good agreement with the experimental value. The reported values of $103.9^{\circ}$ and $104.5^{\circ}$ (Cook et al., 1974; Benedict et al., 1956) for the bond angle are exactly the same as estimated by the MP2(full)/aug-cc-pVDZ and W1U methods respectively. In reality, there must be a water cluster instead of a single water molecule. But to save computational time, here, we are considering a monomer as a representative of the interstellar ice feature. This consideration might propagate some error for our calculated values. But as we are considering some isomeric groups, a well trend of binding energies shall be obtained.

\subsection{Thermodynamics not the key}

The high abundance of methyl formate in different molecular cloud has earned it the name 'interstellar weed'. In the $\mathrm{C}_{2} \mathrm{H}_{4} \mathrm{O}_{2}$ isomeric group, acetic acid is unarguably the most stable isomer while methyl formate ranks second with an energy difference of more than $15 \mathrm{kcal} / \mathrm{mol}$ from both the theoretically predicted and the experimentally measured enthalpy of formation value (Etim and Arunan, 2016, 2017a,b and reference therein). The third most stable isomer on that list is glycolaldehyde. These three isomers have all been detected from different astronomical sources via their rotational transition spectra (Mehringer et al., 1997; Churchwell and Winnewisser, 1975; Hollis et al., 2000). Methyl formate (which is not the most stable isomer of the group) is found to be conspicuously more abundant than both acetic acid and glycolaldehyde in all the molecular clouds where they have been detected. The two known stable isomers of the $\mathrm{C}_{2} \mathrm{H}_{6} \mathrm{O}$ isomeric group; ethanol and dimethyl ether were first detected in the Sagittarius B2 complex and Orion nebula respectively in the 1970s (Zuckerman et al., 1975; Pearson et al., 1997; Snyder et al., 1974). Ethanol is found to be more stable than dimethyl ether with an energy difference of about $12 \mathrm{kcal} / \mathrm{mol}$ from the experimentally reported enthalpy of formation. As such, ethanol should be more abundant than dimethyl ether in the interstellar space. However, the abundance ratio of ethanol and dimethyl ether ranges from 0.3 to 3.0 (Nummelin et al.,
1998, Ikeda et al., 2001, White et al., 2003) with ethanol being more abundant than dimethyl ether in some astronomical observations and the reverse being the case from some other astronomical observations.

Tables 2 and 3 respectively present the binding energies of the hydrogen bonded complexes of the $\mathrm{C}_{2} \mathrm{H}_{4} \mathrm{O}_{2}$ and $\mathrm{C}_{2} \mathrm{H}_{6} \mathrm{O}$ isomers with water at different levels of theory and basis sets considered in this study. Fig. 1 shows the optimized geometries of these complexes at the MP2(full $) / 6-311++G(d, p)$ level of theory. In all the cases, there is an elongation of one of the $\mathrm{O}-\mathrm{H}$ bonds of water monomer which takes part in the hydrogen bond formation from the original $0.959 \AA$ for the water monomer to $0.968,0.965$, $0.970,0.968$, and $0.969 \AA$ for acetic acid, glycolaldehyde, methyl formate, ethanol and dimethyl ether-water complexes respectively at the MP2(full)/6-311++G(d,p) level showing the evidence of hydrogen bond formation in all the systems considered.

Since the binding energies are the keys for controlling the composition of the interstellar grain mantle, it is essential to estimate them in appropriate order to avoid any misleading conclusion. From the binding energies in Table 2, acetic acid-water complex is more strongly bonded followed by glycolaldehyde-water complex while methyl formate-water complex is the least bonded complex. There is an inverse correlation between the binding strength of the complex and the interstellar gas phase abundance of the molecule that is forming the hydrogen bond with water. Methyl formate is more abundant than both acetic acid and glycolaldehyde; glycolaldehyde in turn is more abun-

Table 3

Binding energies for $\mathrm{C}_{2} \mathrm{H}_{6} \mathrm{O}$ isomer complexes with water.

\begin{tabular}{|c|c|c|}
\hline \multirow[t]{2}{*}{ Method } & \multicolumn{2}{|l|}{ BE (Kelvin) } \\
\hline & Ethanol- $\mathrm{H}_{2} \mathrm{O}$ & Dimethyl ether- $\mathrm{H}_{2} \mathrm{O}$ \\
\hline MP2(full)/6-311++G ${ }^{* *}$ & $2566(4527)$ & $2500(4418)$ \\
\hline MP2(full)/aug-cc-pVDZ & $2768(4861)$ & $2717(4776)$ \\
\hline G4 & 2415 (4278) & $2265(4030)$ \\
\hline W1U & $2617(4612)$ & $2566(4527)$ \\
\hline $\begin{array}{c}\text { (Binding energy available from } \\
\text { other studies or estimated) }\end{array}$ & $6260^{\mathrm{a}}$ & $3675^{\mathrm{a}}$ \\
\hline
\end{tabular}

Table 2

Binding energies for $\mathrm{C}_{2} \mathrm{H}_{4} \mathrm{O}_{2}$ isomer complexes with water.

\begin{tabular}{|c|c|c|c|}
\hline \multirow[t]{2}{*}{ Method } & \multicolumn{3}{|l|}{ BE (Kelvin) } \\
\hline & Acetic acid- $\mathrm{H}_{2} \mathrm{O}$ & Glycolaldehyde- $\mathrm{H}_{2} \mathrm{O}$ & Methyl formate- $\mathrm{H}_{2} \mathrm{O}$ \\
\hline MP2(full)/6-311++ $\mathrm{G}^{* *}$ & $3472(6024)$ & $2768(4861)$ & $1635(2989)$ \\
\hline G4 & $4831(8268)$ & $2667(4694)$ & $2164(3863)$ \\
\hline W1U & $4680(8019)$ & $2818(4944)$ & 2415 (4278) \\
\hline (Binding energy available from other studies or estimated) $^{\mathrm{a}}$ & $6300^{\mathrm{a}}$ & $6680^{\mathrm{a}}$ & $5200^{\mathrm{a}}$ \\
\hline
\end{tabular}

\footnotetext{
${ }^{\text {a }}$ Garrod, 2013, Bracketed values are scaled as $(289.019+(1.65174 \times$ Calculated BE $))$.
} 
dant than acetic acid in the different molecular clouds where they have been detected (Mehringer et al., 1997; Remijan et al., 2002; Remijan et al., 2003; Hollis et al., 2001; Hollis et al., 2004a,b; Cazaux et al., 2003). Thus, the higher the binding strength of a complex with water, the lower the interstellar abundance of the molecule as compared to its counterparts with lower binding strength. This is because the more strongly the molecule is bonded to the surface of the interstellar dust grains; the more a greater part of it is being attached to the surface of the interstellar dust grains thereby reducing its abundance. In contrary to our calculated values, other estimation remains. For example, Garrod (2013) estimated that among this isomeric group, glycolaldehyde is bonded more strongly than methyl formate.

Ethanol is more strongly bonded to the surface of the interstellar dust grains as evident in binding energy of the ethanol-water complex as compared to the dimethyl ether-water complex (Table 3). Garrod (2013) also used higher binding energy of ethanol compare to dimethyl ether. From the above analogy, dimethyl ether should be more abundant than ethanol and this has been observed in a few cases. The high abundance of ethanol over dimethyl ether in many cases points to gas phase reactions as the dominant formation processes in such cases where the effect of hydrogen bonding is drastically reduced, thus the abundance of ethanol is not affected as in the case of interstellar dust grains formation process.

\subsection{Delayed observation of the most stable isomers}

In many of the known interstellar isomeric species, the most stable isomer which is probably the most abundant isomer in the interstellar medium has not always been the first isomer to be detected. Though $\mathrm{HCN}$ was detected before $\mathrm{HNC}, \mathrm{MgNC}$ before $\mathrm{MgCN}, \mathrm{HNCS}$ before HSCN, $\mathrm{HC}_{3} \mathrm{~N}$ before $\mathrm{HC}_{2} \mathrm{NC}$ and $\mathrm{HNC}_{3}$, methyl cyanide before methyl isocyanide and ketenimine, acetaldehyde before vinyl alcohol and ethylene oxide, this trend goes on and on (Zuckerman et al., 1972, Ziurys et al., 1995; Kawaguchi et al., 1993; Halfen et al., 2009; Frerking et al., 1979; Fourikis et al., 1974; Gilmore et al., 1976; Turner and Apponi, 2001; Dickens et al., 1997; Etim and Arunan, 2016). But this is not true as interstellar chemistry is far away from the thermodynamic equilibrium. For instance, Loison et al., 2016 studied $\mathrm{C}_{3} \mathrm{H}_{2} \mathrm{O}$ isomeric group and found that though propadienone is the most stable species, only cyclopropenone and propynal have been detected in the ISM.

Propanoic acid $\left(\mathrm{C}_{3} \mathrm{H}_{6} \mathrm{O}_{2}\right.$ isomeric species $)$ and propan2-ol and propanol $\left(\mathrm{C}_{3} \mathrm{H}_{8} \mathrm{O}\right.$ isomeric species $)$ are the most stable isomers in their respective isomeric groups. Whereas other less stable isomers have been observed in these groups but none of these most stable isomers have been observed. The binding energies of the hydrogen bonded complexes with water formed by these isomers (most stable and the astronomically observed) are shown in Tables 4 $\left(\mathrm{C}_{3} \mathrm{H}_{6} \mathrm{O}_{2}\right.$ isomers $)$ and $5\left(\mathrm{C}_{3} \mathrm{H}_{8} \mathrm{O}\right.$ isomers $)$. Ethyl formate and methyl acetate from the $\mathrm{C}_{3} \mathrm{H}_{6} \mathrm{O}_{2}$ isomeric group have been detected in the interstellar space (Belloche et al., 2009; Tercero et al., 2013). While ethyl methyl ether is the only astronomically observed isomer of the $\mathrm{C}_{3} \mathrm{H}_{8} \mathrm{O}$ group (Fuchs et al., 2005). Fig. 2 shows the optimized geometries of the $\mathrm{C}_{3} \mathrm{H}_{6} \mathrm{O}_{2}$ and $\mathrm{C}_{3} \mathrm{H}_{8} \mathrm{O}$ isomeric species considered here.

At the MP2(full)/6-311++G (d,p) level, the $\mathrm{O}-\mathrm{H}$ bond distance of the water monomer that is forming complex with the interstellar molecule is extended from the normal bond length $(0.959 \AA)$ into the range of $0.965-0.969 \AA$ for the hydrogen bonded complexes shown in Fig. 2.

Propanoic acid is the most stable isomer of the $\mathrm{C}_{3} \mathrm{H}_{6} \mathrm{O}_{2}$ isomeric group followed by ethyl formate while methyl acetate comes third in the energy scale. From the binding energies presented in Table 4, propanoic-water complex is more strongly bonded to the surface of the interstellar dust grains as compared to the ethyl formate-water and methyl acetate-water complexes. Garrod (2013) also considered the similar trends between the binding energies of these three species. This clearly shows that a greater portion of propanoic acid is attached to the surface of the interstellar dust grains via hydrogen bonding thereby reducing its available interstellar gas phase abundance thus the delay in its astronomical detection. In the $\mathrm{C}_{3} \mathrm{H}_{8} \mathrm{O}$ isomeric group, propan-2-ol is the most stable, followed by propanol while ethyl methyl ether is the least stable isomer of the group ${ }^{1}$. As it is evident in Table 5, the isomers that have not been observed are more strongly bonded to the surface of the interstellar dust grains than the only observed isomer of the group. This accounts for the delay in the astronomical observations of these isomers as their abundances are

Table 4

Binding energies for $\mathrm{C}_{3} \mathrm{H}_{6} \mathrm{O}_{2}$ isomer complexes with water.

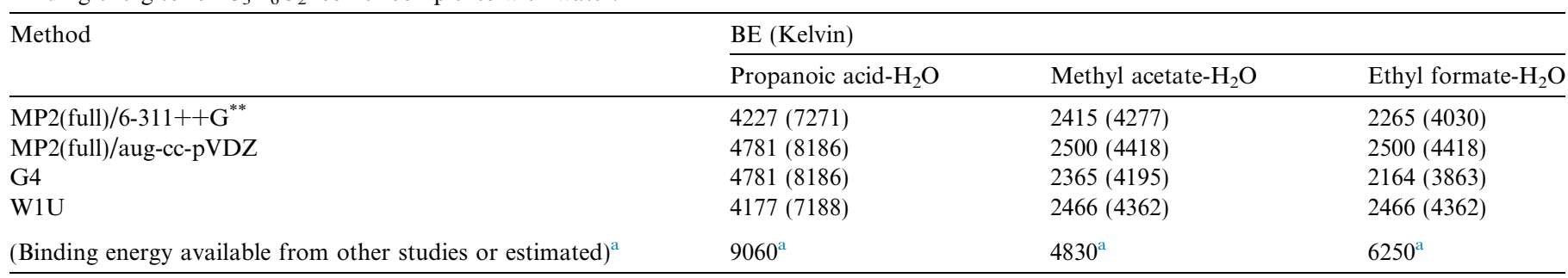

${ }^{a}$ Garrod, 2013, Bracketed values are scaled as $(289.019+(1.65174 \times$ Calculated BE $))$. 


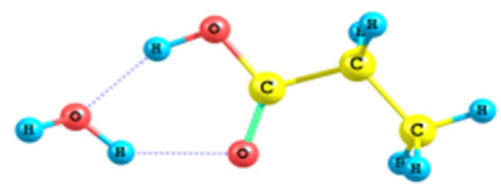

Propanoic acid-water complex

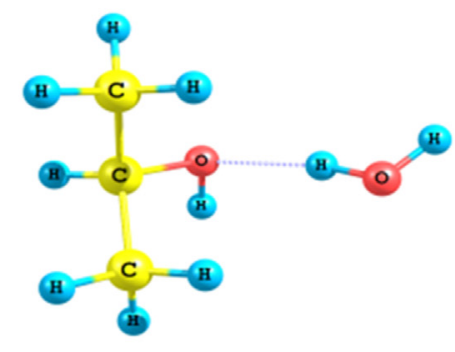

Propan-2-ol-water complex

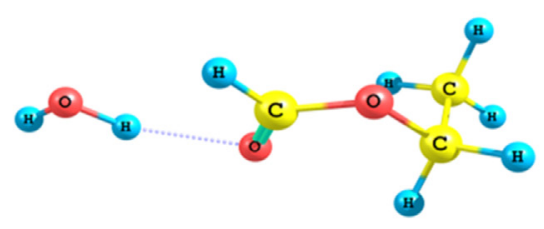

Ethyl formate-water complex

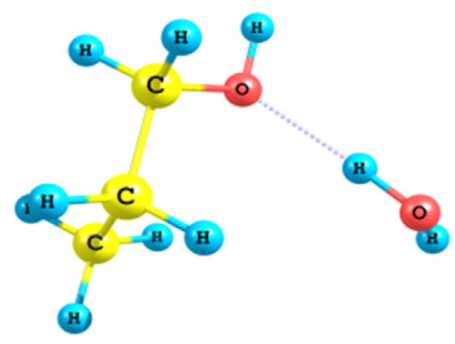

Propanol-water complex

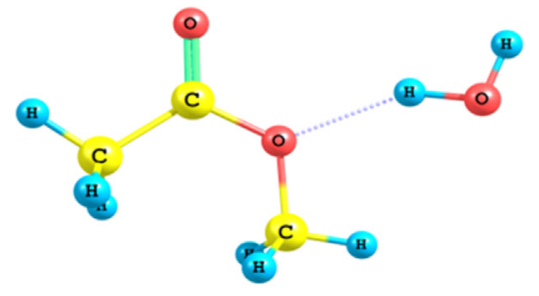

Methyl acetate-water complex

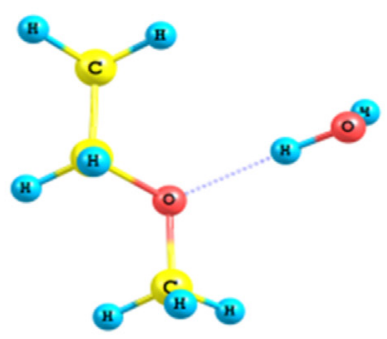

Ethyl methyl ether-water complex

Fig. 2. Optimized structures of the hydrogen bonded $\mathrm{C}_{3} \mathrm{H}_{6} \mathrm{O}_{2}$ and $\mathrm{C}_{3} \mathrm{H}_{8} \mathrm{O}$ isomer complexes with water.

Table 5

Binding energies for $\mathrm{C}_{3} \mathrm{H}_{8} \mathrm{O}$ isomer complexes with water.

\begin{tabular}{|c|c|c|c|}
\hline \multirow[t]{2}{*}{ Method } & \multicolumn{3}{|l|}{ BE (Kelvin) } \\
\hline & Propan-2-ol- $\mathrm{H}_{2} \mathrm{O}$ & Propanol- $\mathrm{H}_{2} \mathrm{O}$ & Ethyl methyl ether- $\mathrm{H}_{2} \mathrm{O}$ \\
\hline MP2(full)/6-311++G ${ }^{* *}$ & 2717 (4777) & 2617 (4612) & $2516(4445)$ \\
\hline MP2(full)/aug-cc-pVDZ & $2969(5193)$ & $2868(5026)$ & $2717(4776)$ \\
\hline G4 & $2516(4444)$ & $2415(4278)$ & $2365(4195)$ \\
\hline $\mathrm{W} 1 \mathrm{U}$ & $2768(4696)$ & $2667(4694)$ & $2617(4612)$ \\
\hline (Binding energy available from other studies or estimated) $^{\mathrm{a}}$ & $4260^{\mathrm{b}}$ & $7190^{\mathrm{d}}$ & $5495^{\mathrm{a}}$ \\
\hline
\end{tabular}

Bracketed values are scaled as $(289.019+(1.65174 \times$ Calculated BE $))$.

${ }^{a}$ Taquet et al. (2016).

${ }^{b}$ Binding energy estimated by adding the binding energies of two $2 \mathrm{CH}_{3}$ and $\mathrm{CHOH}$.

${ }^{d}$ Binding energy estimated by adding the binding energies of $\mathrm{C}_{2} \mathrm{H}_{5}$ and $\mathrm{CH}_{2} \mathrm{OH}$.

reduced due to this interstellar hydrogen bonding as discussed in the previous cases. Estimated binding energies of $\mathrm{C}_{3} \mathrm{H}_{8} \mathrm{O}$ isomeric group are also presented in Table 5. Taquet et al. (2016) estimated the binding energy of ethyl methyl ether to be $5495 \mathrm{~K}$. Binding energy of the propan2-ol is estimated by adding the binding energies of two $\mathrm{CH}_{3}$ molecules along with $\mathrm{CHOH}$. Binding energy of propanol is estimated by summing up the binding energies of $\mathrm{C}_{2} \mathrm{H}_{5}$ and $\mathrm{CH}_{2} \mathrm{OH}$. Binding energies of these radicals are mainly considered from Garrod (2013). If these binding energies are not listed in Garrod (2013), we consider it from Hasegawa et al. (1992). Thus from the estimated list of binding energies of this isomeric species, propanol is found to be possess the strongest barrier.

\subsection{Unsuccessful observations: Amino acids}

Amino acids are water-soluble organic molecules containing both the amine $\left(-\mathrm{NH}_{2}\right)$ and the carboxyl
$(-\mathrm{COOH})$ groups. Interestingly, from the functional groups alone, the amino acids contain the four most important biogenic elements; carbon, hydrogen, oxygen and nitrogen. The class of amino acids in which both the functional groups are attached to the same carbon atom called the $\alpha$-carbon atom, are of utmost biological importance being the building blocks of proteins. As a result of this, the search for amino acid in the interstellar medium has been the subject of interest. In particular, glycine; the simplest member of the $\alpha$-amino acids has attracted the attention of researchers globally. All the searches for glycine in the interstellar medium have not yielded any successful detection of this molecule (Kuan et al., 2003; Snyder et al., 2005; Jones et al., 2007; Cunningham et al., 2007). Though there has not been any successful detection of the amino acid in the interstellar medium, about 80 of them have been identified in meteorites found on Earth and the origin of these compounds fond in meteorites are often traced to the interstellar medium; thus linking the interstel- 
lar medium and the chemical composition of the meteorites (Elsila et al., 2007; Botta and Bada, 2002).

Glycine belongs to the $\mathrm{C}_{2} \mathrm{H}_{5} \mathrm{NO}_{2}$ isomeric group. The three most stable isomers of this group are methyl carbamic acid, glycine and methyl carbamate respectively. Fig. 3 shows the optimized structures of these isomers complexes with water while Table 6 gives the binding energies of these complexes. The elongation of the $\mathrm{O}-\mathrm{H}$ bond length of water is also observed as in the previous cases. From Table 6, the most stable isomer of the $\mathrm{C}_{2} \mathrm{H}_{5} \mathrm{NO}_{2}$ group; methyl carbamic acid is found to be more strongly bonded to the surface of the interstellar dust grains followed by glycine (the second most stable isomer of the group) and lastly by methyl carbamate. Though methyl carbamic acid has not been searched for possibly because of the lack of its rotational transitions the other two isomers have been searched for in different astronomical sources. Whereas there has been no successful detection of glycine, but methyl carbamate has been tentatively observed in the molecular cloud (Demyk et al., 2004). The high bonding strength of glycine to the surface of the interstellar dust grains can easily been seen as having a direct effect on its abundance (reduction in abundance) thus contributing to it unsuccessful astronomical observations. Estimated binding energy values are also shown in Table 6. These energy values are not in line with our calculated values. Garrod (2013) estimated very high binding energy of glycine $(10,100 \mathrm{~K})$. From the existing values, we estimate the binding energies of methyl carbamic acid by considering the addition of $\mathrm{CH}_{3} \mathrm{NH}$ and $\mathrm{COOH}$ and binding energy of Methyl carbamate by the addition of $\mathrm{CH}_{3}, \mathrm{CO}_{2}$ and $\mathrm{NH}_{2}$.

\subsection{Potential candidate for astronomical observation: Ketenes}

As discussed under the delayed observation of the most stable isomers; the most stable isomer is mostly observed before the other less stable isomers. The ketenes (ketene, methylene ketene and methyl ketene) are found to be the most stable isomers in their respective isomeric groups (Etim and arunan 2016), in each of these groups the other less stable isomers have been detected whereas ketene has only been detected in only one group $\left(\mathrm{C}_{2} \mathrm{H}_{2} \mathrm{O}\right.$ isomeric group). Abundances of the most stable isomer may be affected by interstellar hydrogen bonding thereby reducing its abundance, thus making its astronomical observation difficult. In testing the effect of interstellar hydrogen bonding among the ketenes, we subject ketenes and the observed isomer in each group to form hydrogen bonded complex with water.

Fig. 4 shows the optimized structures of the $\mathrm{C}_{2} \mathrm{H}_{2} \mathrm{O}$, $\mathrm{C}_{3} \mathrm{H}_{2} \mathrm{O}$ and $\mathrm{C}_{3} \mathrm{H}_{4} \mathrm{O}$ complexes with water considered in this study. Tables 7-9 contain the binding energies of the $\mathrm{C}_{3} \mathrm{H}_{4} \mathrm{O}, \mathrm{C}_{3} \mathrm{H}_{2} \mathrm{O}$ and $\mathrm{C}_{2} \mathrm{H}_{2} \mathrm{O}$ complexes with water respectively. The formation of hydrogen bond between the water monomer and the interstellar molecule is observed in all the cases via the elongation of one of the $\mathrm{O}-\mathrm{H}$ bonds of water monomer which takes part in the hydrogen bond forma-
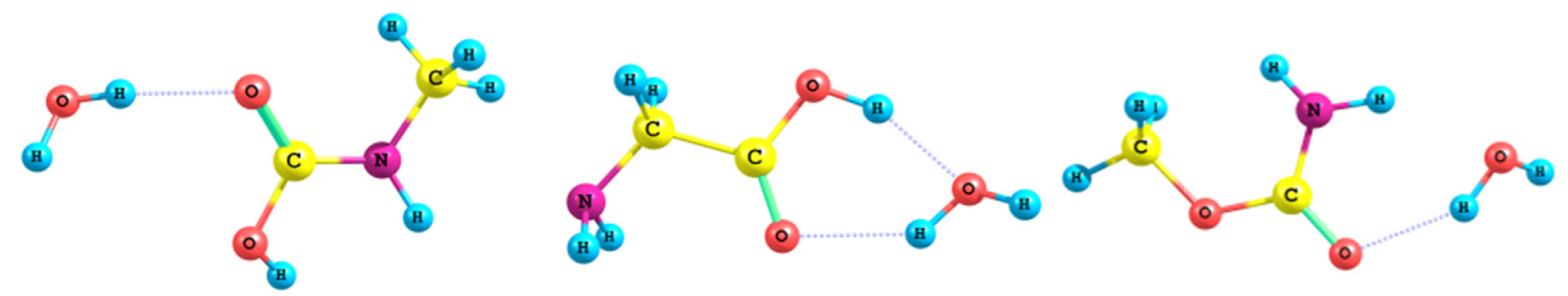

\section{Methyl carbamic acid-water complex Glycine-water complex Methyl carbamate-water complex}

Fig. 3. Optimized structures of the hydrogen bonded $\mathrm{C}_{2} \mathrm{H}_{5} \mathrm{NO}_{2}$ complexes with water.

Table 6

Binding energies for $\mathrm{C}_{2} \mathrm{H}_{5} \mathrm{NO}_{2}$ isomer complexes with water.

\begin{tabular}{|c|c|c|c|}
\hline \multirow[t]{2}{*}{ Method } & \multicolumn{3}{|l|}{ BE (Kelvin) } \\
\hline & Methyl carbamic acid- $\mathrm{H}_{2} \mathrm{O}$ & Glycine- $\mathrm{H}_{2} \mathrm{O}$ & Methyl carbamate- $\mathrm{H}_{2} \mathrm{O}$ \\
\hline MP2(full)/6-311++G ${ }^{* *}$ & 4177 (7188) & $2415(4277)$ & $1560(2865)$ \\
\hline MP2(full)/aug-cc-pVDZ & $4730(8101)$ & $2566(4527)$ & $1711(3115)$ \\
\hline G4 & $4277(7353)$ & $2566(4527)$ & $1560(2865)$ \\
\hline W1U & $4630(7936)$ & $2617(4611)$ & $1661(3032)$ \\
\hline (Binding energy available from other studies or estimated) ${ }^{\mathrm{a}}$ & $6680^{\mathrm{b}}$ & $10100^{\mathrm{a}}$ & $7710^{\mathrm{d}}$ \\
\hline
\end{tabular}

Bracketed values are scaled as $(289.019+(1.65174 \times$ Calculated BE $))$.

${ }^{a}$ Garrod (2013).

${ }^{b}$ Binding energy estimated by adding the binding energies of $\mathrm{CH}_{3} \mathrm{NH}+\mathrm{COOH}$.

${ }^{d}$ Binding energy estimated by adding the binding energies of $\mathrm{CH}_{3}+\mathrm{CO}_{2}+\mathrm{NH}_{2}$. 
tion from the original $0.959 \AA$ for the water monomer to $0.966,0.961,0.965,0.961,0.960$ and $0.961 \AA$ for propenal, methyl ketene, propynal, methylene ketene, ethynol and ketene-water complexes respectively at the MP2(full)/6$311++G(d, p)$ level. In ketene-water complex where the water molecule acts as hydrogen bond acceptor rather than donor as in the previous cases, there is an elongation of both the $\mathrm{O}-\mathrm{H}$ bonds of the water monomer. This is pictured in Fig. 4.

From the binding energies of $\mathrm{C}_{2} \mathrm{H}_{2} \mathrm{O}, \mathrm{C}_{3} \mathrm{H}_{2} \mathrm{O}$ and $\mathrm{C}_{3} \mathrm{H}_{4} \mathrm{O}$ complexes with water presented in Tables 7-9 respectively, it is crystal clear that the ketenes are less strongly bonded to the surface of the interstellar dust grains as compared to their respective isomers that have

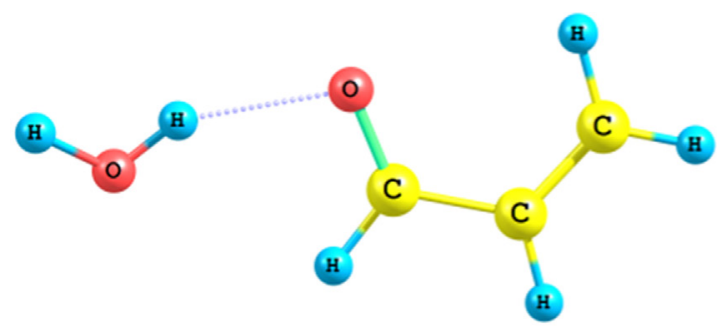

Propenal-water complex
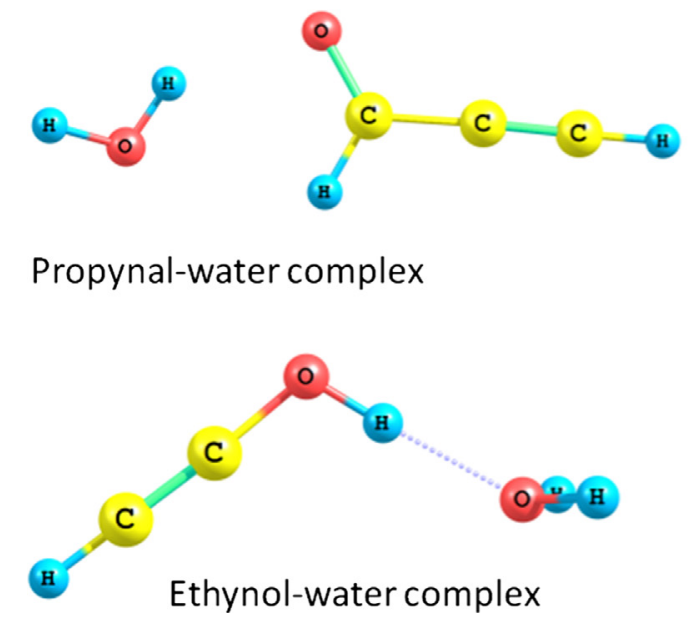

Fig. 4. Optimized structures of the hydrogen bonded $\mathrm{C}_{3} \mathrm{H}_{4} \mathrm{O}, \mathrm{C}_{3} \mathrm{H}_{2} \mathrm{O}$ and $\mathrm{C}_{2} \mathrm{H}_{2} \mathrm{O}$ complexes with water.

Table 7

Binding energies for propenal and methyl ketene-water complexes.

\begin{tabular}{|c|c|c|}
\hline \multirow[t]{2}{*}{ Method } & \multicolumn{2}{|l|}{ BE (Kelvin) } \\
\hline & Propenal- $\mathrm{H}_{2} \mathrm{O}$ & Methyl ketene- $\mathrm{H}_{2} \mathrm{O}$ \\
\hline MP2(full)/6-311++G ${ }^{* *}$ & $2315(4112)$ & 1057 (2034) \\
\hline MP2(full)/aug-cc-pVDZ & $2617(4612)$ & $1409(2616)$ \\
\hline G4 & 2204 (3929) & 1208 (2284) \\
\hline W1U & $2516(4444)$ & $1308(2449)$ \\
\hline 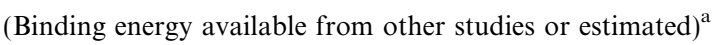 & $5530^{\mathrm{b}}$ (as like methanol) & $3160^{\mathrm{d}}\left(\mathrm{C}_{2} \mathrm{H}_{4}+\mathrm{CO}\right)$ \\
\hline
\end{tabular}

Bracketed values are scaled as $(289.019+(1.65174 \times$ Calculated BE) $)$.

${ }^{\mathrm{b}}$ Considered same as methanol.

${ }^{d}$ Binding energy estimated by adding the binding energies of $\mathrm{C}_{2} \mathrm{H}_{4}$ and $\mathrm{CO}$.

been astronomically observed (Irvine et al., 1988; Turner, 1977). Estimated values, which are also pointed out in Tables 7-9 depicts the similar trend. That ketenes are thermodynamically the most stable isomers in their respective isomeric groups, they are not affected by interstellar hydrogen bonding as compared to their isomers that have been detected and that a ketene molecule been astronomically detected in the interstellar medium, we therefore propose other ketene molecules as potential candidates for astronomical observations especially methyl and methylene ketenes considered in this study.

It is worth mentioning here that a ketenyl radical; $\mathrm{HCCO}$ has recently been detected in ISM in line with our prediction (Agúndez et al., 2015).

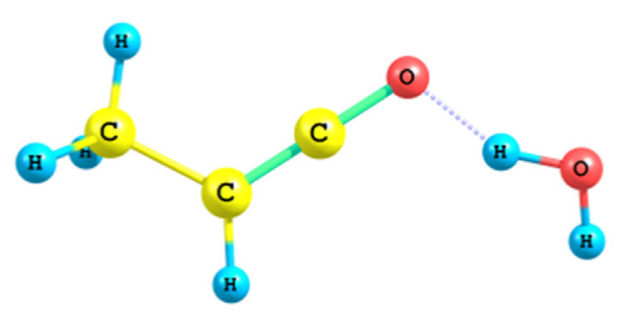

Methyl ketene-water complex
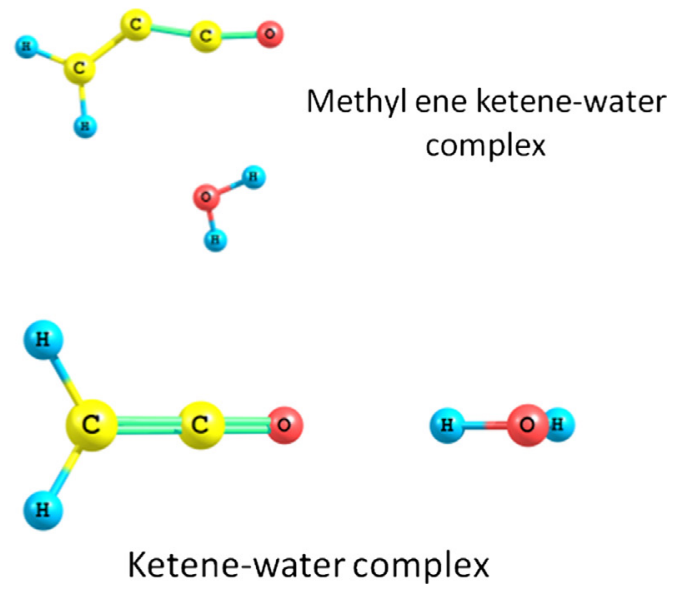

\footnotetext{
-
} 
Table 8

Binding energies for propynal and methylene ketene-water complexes.

\begin{tabular}{|c|c|c|}
\hline \multirow[t]{2}{*}{ Method } & \multicolumn{2}{|l|}{ BE (Kelvin) } \\
\hline & Propynal- $\mathrm{H}_{2} \mathrm{O}$ & Methylene ketene- $\mathrm{H}_{2} \mathrm{O}$ \\
\hline $\mathrm{MP} 2($ full $) / 6-311++\mathrm{G}^{* *}$ & $2114(3780)$ & $1308(2449)$ \\
\hline MP2(full)/aug-cc-pVDZ & $2415(4278)$ & $1500(2766)$ \\
\hline G4 & $2063(3696)$ & $1006(1950)$ \\
\hline W1U & $2566(4527)$ & 1107 (2117) \\
\hline$\left(_{\text {Binding energy available from other studies or estimated) }}{ }^{\mathrm{a}}\right.$ & $3060^{\mathrm{b}}\left(\mathrm{C}_{2} \mathrm{H}+\mathrm{H}_{2} \mathrm{CO}-\mathrm{H}\right)$ & $2966^{\mathrm{b}}\left(\mathrm{CH}_{2}+\mathrm{C}_{2} \mathrm{O}\right)$ \\
\hline
\end{tabular}

Bracketed values are scaled as $(289.019+(1.65174 \times$ Calculated BE $))$.

${ }^{\mathrm{d}}$ Binding energy estimated by adding the binding energies of $\mathrm{CH}_{2}$ and $\mathrm{C}_{2} \mathrm{O}$.

${ }^{b}$ Binding energy estimated by adding the binding energies of $\mathrm{C}_{2} \mathrm{H}$ and $\mathrm{H}_{2} \mathrm{CO}$ and deducting binding energy of $\mathrm{H}$.

Table 9

Binding energies for ethynol and ketene-water complexes.

\begin{tabular}{|c|c|c|}
\hline \multirow[t]{2}{*}{ Method } & \multicolumn{2}{|l|}{ BE (Kelvin) } \\
\hline & Ethynol- $\mathrm{H}_{2} \mathrm{O}$ & Ketene- $\mathrm{H}_{2} \mathrm{O}$ \\
\hline $\mathrm{MP} 2($ full $) / 6-311++\mathrm{G}^{* *}$ & $3849(6646)$ & $905(1783)$ \\
\hline MP2(full)/aug-cc-pVDZ & $4232(7279)$ & $1006(1950)$ \\
\hline G4 & $4428(7602)$ & $956(1868)$ \\
\hline W1U & $4378(7520)$ & 1107 (2117) \\
\hline (Binding energy available from other studies or estimated) $^{\mathrm{a}}$ & $2414^{\mathrm{d}}$ & 2200 \\
\hline
\end{tabular}

Bracketed values are scaled as $(289.019+(1.65174 \times$ Calculated BE $))$.

${ }^{\mathrm{d}}$ Binding energy estimated by adding the binding energies of $\mathrm{CH}$ and $\mathrm{HOC}$.

\subsection{Detecting weakly bound complexes in the ISM?}

From the foregoing discussions, the existence of weakly bound complexes in the interstellar medium is well established. But these weakly bound complexes that are formed in ISM, are they detectable? The interstellar molecular species are observed in the gas phase (both the ones that are formed on the surface of the interstellar dust grains and those that are gas phase products). During the warm-up phase associated with formation of stars, the molecular species that are formed on the surface of the interstellar

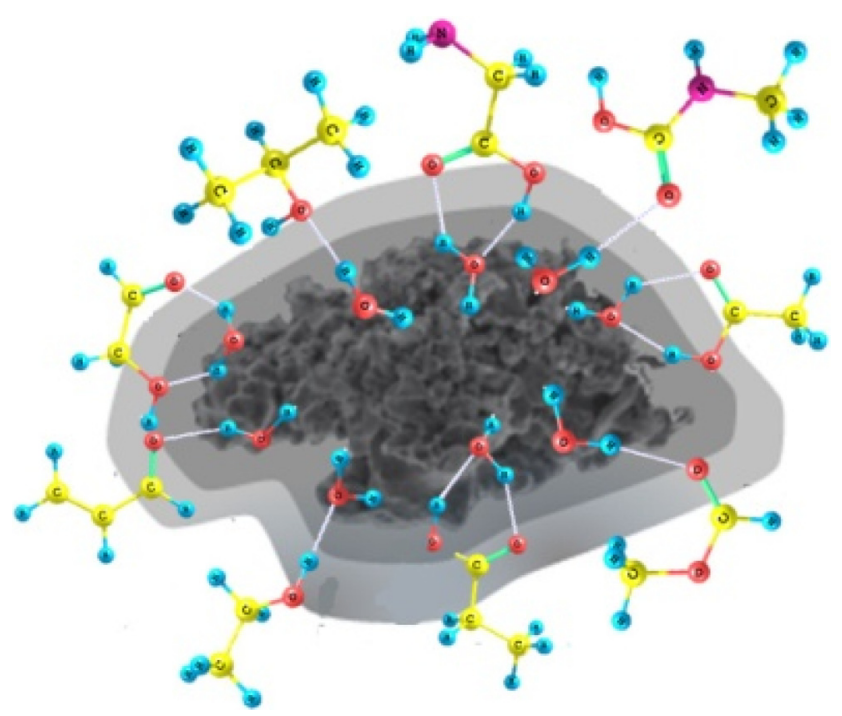

Fig. 5. Schematic picture of the effect of interstellar hydrogen bonding. dust grains will be desorbed. The desorbed species will enter the gas phase where they are normally detected; codesorption of these molecules and water in the form of weakly bound complexes or clusters could be highly possible. Also, the conditions in the terrestrial laboratories where weakly bound complexes are observed are similar to the conditions in ISM. In addition, the high binding energies of the complexes as shown in Tables 2-9 imply that these complexes are detectable in ISM. Thus, it suffices to say that weakly bound complexes are present and detectable in the interstellar medium.

Fig. 5 paints a picture of the effect of interstellar hydrogen bonding. It shows how the interstellar molecules are getting bonded to the water molecule on the surface on the interstellar dust grains, thus causing a greater part of these interstellar molecules to be attached to the surface of the interstellar dust grains. This leads in the low abundances observed for these molecules and may be the reason for unsuccessful observations of others at low temperature.

The use of different high level quantum ab initio methods in computing the binding energies of these complexes test the reliability and consistency of the results in all the cases considered.

\section{Conclusion}

The present study reports the extensive study of the existence and effects of interstellar hydrogen bonding. The binding energies of the hydrogen bonded complexes of interstellar molecules with water monomer obtained from high level quantum chemical simulations show a direct 
relationship between the binding energies of some species (whose formation is mainly controlled by the ice phase reactions) and the interstellar abundances of the molecules. From the relationship, the higher the binding energy of the interstellar molecule bonded with water, the lower its interstellar abundance as compared to its counterparts with lower binding energies. This is because the stronger the molecule is being bonded to the surface of the interstellar dust grains; the more a greater portion of it is being attached to the surface of the interstellar dust grains, thereby reducing its gas phase abundance. Available interstellar observations data confirms this. Interstellar hydrogen bonding accounts for the deviations from thermodynamically controlled processes, the delay in detecting the most stable isomers whose less stable counterparts have been detected, the difficulty in observing amino acids (e.g. glycine). From this and our previous studies, we propose methyl and methylene ketenes as potential candidates for astronomical observations. The acids are more strongly bonded to the surface of the interstellar dust grains than their corresponding isomers in all the cases observed here. Could this point to the detectability of acid-water complexes in the interstellar medium soon? Apart from water and other major components of the interstellar ice, the formation of hydrogen bonded complexes between these components and other molecules suggests the presence of other minor components in the interstellar ice like acetic acid and other strongly hydrogen bonded molecules. This is worth investigating.

\section{Acknowledgement}

EEE acknowledges a research fellowship from the Indian Institute of Science, Bangalore. AD, PG and SKC acknowledge an ISRO Respond project (Grant No. ISRO/RES/2/402/16-17).

\section{Appendix A. Supplementary material}

Supplementary data associated with this article can be found, in the online version, at https://doi.org/10.1016/j. asr.2018.03.003.

\section{References}

Agúndez, M., Cernicharo, J., Guélin, M., 2015. A\&A 577, L5.

Belloche, A., Garrod, R.T., Müller, H.S.P., Menten, K.M., Comito, C., Schilke, P., 2009. A\&A 499, 215-232.

Benedict, W.S., Gailan, N., Plyler, E.K., 1956. J. Chem. Phys. 24, 11391165.

Biham, O., Furman, I., Pirronelo, V., Vidali, G., 2001. ApJ 553, 595.

Botta, O., Bada, J.L., 2002. Surveys Geophys. 23, 411-467.

Boys, S.F., Bernardi, F., 1970. Mol. Phys 19, 553-566.

Chakrabarti, S.K., Das, A., Acharyya, K., Chakrabarti, S., 2006. A\&A $522,74$.

Chandrasekhar, J., Andrade, J.G., Schleyer, P.V.R., 1981. JACS 103, 5609-5612.

Churchwell, E., Winnewisser, G., 1975. A\&A 45, 229-231.
Cazaux, S., Tielens, A.G.G.M., Ceccarelli, C., Castets, A., Wakelam, V., Caux, E., Parise, B., Teyssier, D., 2003. ApJ 593, L51-L55.

Cook, R.L., de Lucia, F.C., Helminger, P., 1974. J. Mol. Spectrosc 53, $62-$ 76.

Curtiss, L.A., Raghavachari, K., Redfern, P.C., Rassolov, V., Pople, J.A., 1998. J. Chem. Phys. 109, 7764-7776.

Curtiss, L.A., Redfern, P.C., Raghavachari, K., 2007a. J. Chem. Phys 126, 084108-12.

Curtiss, L.A., Redfern, P.C., Raghavachari, K., 2007b. J. Chem. Phys 127, 124105-8.

Cunningham, M.R., Jones, P.A., Godfrey, P.D., Cragg, D.M., Bains, I., Burton, M.G., Calisse, P., Crighton, N.H.M., Curran, S.J., Davis, T. M., 2007. MNRAS 376, 1201-1210.

Das, A., Acharyya, K., Chakrabarti, S.K., 2010. MNRAS 409, 789.

Das, A., Chakrabarti, S.K., 2011. MNRAS 418, 545.

Das, A., Sahu, D., Majumdar, L., Chakrabarti, S.K., 2016. MNRAS 455, 540.

Dickens, J.E., Irvine, W.M., Ohishi, M., Ikeda, M., Ishikawa, S., Nummelin, A., Hjalmarson, A., 1997. ApJ 489, 753-757.

Draine, B.T., 2003. Annu. Rev. Astron. Astrophys 41, 241-289.

Demyk, K., Wlodarczak, G., Dartois, E., 2004. In: Combes, F, Barret, D., Contini, T., Meynadier, F., Pagani, L. (Eds.), Semaine de l'Astrohysique Francaise, SF2A-2004, EDP-Sciences, Les Ulis, p. 493.

Elsila, E., Dworkin, J.P., Bernstein, M.P., Martin, M.P., Sandford, S.A., 2007. ApJ 660, 911-918.

Etim, E.E., Gorai, P., Das, A., Chakrabarti, S.K., Arunan, E., 2016. ApJ 832, 144.

Etim, E.E., Arunan, E., 2016. EPJ-P 131, 448. https://doi.org/10.1140/ epjp/i2016-16448-0.

Etim, E.E., Arunan, E., 2017a. Astrophys. Space Sci. 362, 4.

Etim, E.E., Arunan, E., 2017b. Adv. Space Res. 59 (4), 1161-1171.

Etim, E.E., Gorai, P., Das, A., Arunan, E., 2017. EPJD 71, 86.

Frisch, M.J., Head-Gordon, M., Pople, J.A., 1990. Chem. Phys. Lett. 166, 281-289.

Frisch, M.J., Trucks, G.W., Schlegel, H.B., Scuseria, G.E., Robb, M.A., Cheeseman, J.R., Scalmani, G., Barone, V., Mennucci, B., Petersson, G.A., Gaussian 09, 2009, revision D.01;Gaussian, Inc., Wallingford, CT.

Fraser, H.J., McCoustra, M.R.S., Williams, D.A., 2002. Astron Geophys. 43 (2), 10-18.

Frerking, M.A., Linke, R.A., Thaddeus, P., 1979. ApJ 234, 143-145.

Fuchs, G.W., Fuchs, U., Giesen, T.F., Wyrowski, F., 2005. A\&A 444, 521-530.

Fourikis, N., Sinclair, M.W., Robinson, B.J., Godfrey, P.D., Brown, R. D., 1974. AuJPh 27, 425-430.

Garrod, R.T., 2013. ApJ 765, 50.

Gibb, E., Nummelin, A., Irvine, W.M., Whittet, D.C.B., Bergman, P., 2000. ApJ 545, 309-326.

Gibb, E.L., Whittet, D.C.B., Boogert, A.C.A., Tielens, A.G.G.M., 2004. ApJS 151, 35-73.

Gilmore, W., Morris, M., Palmer, P., Johnson, D.R., Lovas, F.J., Turner, B.E., Zuckerman, B., 1976. ApJ 204, 43-46.

Gorai, P., Das, A., Das, A., Etim, E.E., Chakrabarti, S.K., 2017. ApJ 836, 70.

Hasegawa, T., Herbst, E., Leung, C.M., 1992. ApJ 82, 167.

Halfen, D.T., Ziurys, L.M., Brünken, S., Gottlieb, C.A., McCarthy, M.C., Thaddeus, P., 2009. ApJ 702, 124-127.

Hariharan, P.C., Pople, J.A., 1973. Theor. Chim. Acta 28, 213-222.

Head-Gordon, M., Pople, J.A., Frisch, M.J., 1988. Chem. Phys. Lett. 153 (6), 503-506.

Hollis, J.M., Vogel, S.N., Snyder, L.E., Jewell, P.R., Lovas, F.J., 2001. ApJ 554, L81-L85.

Hollis, J.M., Jewell, P.R., Lovas, F.J., Remijan, A., 2004a. ApJ 613, L45L48.

Hollis, J.M., Lovas, F.J., Jewell, P.R., 2000. ApJ 540, 107-110.

Hollis, J.M., Jewell, P.R., Lovas, F.J., Remijan, A., Møllendal, H., 2004 b. ApJ 610, 21-24.

Ikeda, A., Nakao, Y., Sato, H., Sakaki, S., 2007. JPC-A 111, 7124-7132. 
Ikeda, M., Ohishi, M., Dickens, J.E., Bergman, P., Hjalmarson, A., Irvine, W.M., 2001. ApJ 560, 792-805.

Irvine, W.M., Brown, R.D., Craig, D.M., Friberg, P., Godfrey, P.D., Kaifu, N., Matthews, H.E., Ohishi, M., Suzuki, H., Takeo, H., 1988. ApJ 335, 89-93.

Jones, P.A., Cunningham, M.R., Godfrey, P.D., Cragg, D.M., 2007. MNRAS 374, 579-589.

Kawaguchi, K., Kagi, E., Hirano, T., Takano, S., Saito, S., 1993. ApJ 406, 39-42.

Kuan, Y.-J., Charnley, S.B., Huang, H.-C., Tseng, W.-L., Kisiel, Z., 2003. ApJ 593, 848-867.

Loison, J.-C., Agundez, M., Macrcelino, N., Wakelam, V., 2016. MNRAS 456, 4101-4110.

Møller, C., Plesset, M.S., 1943. Phys. Rev. 46, 618-622.

Martin, J.M.L., de Oliveira, G., 1999. J. Chem. Phys. 111, 1843-1856.

Mehringer, D.M., Snyder, L.E., Miao, Y., Lovas, F., 1997. ApJ 480, 7174.

Nummelin, A., Bergman, P., Hjalmarson, A., Friberg, P., Irvine, W.M., Millar, T.J., Ohishi, M., Saito, S.A., 1998. ApJS 117, 427-529.

Parthiban, S., Martin, J.M.L., 2001. J. Chem. Phys. 114, 6014-6029.

Park, Y.C., Lee, J.S., 2007. Bull. Korean Chem. Soc. 28 (3), 386-390.

Pearson, J.C., Sastry, K.V.L.N., Herbst, E., De Lucia, F.C., 1997. ApJ 480, 420-431.

Remijan, A., Snyder, L.E., Liu, S.Y., Mehringer, D., Kuan, Y.J., 2002. ApJ 576, 264-273.

Remijan, A., Snyder, L.E., Friedel, D.N., Liu, S.Y., Shah, R.Y., 2003. ApJ 590, 314-332.
Snyder, L.E., Buhl, D., Schwartz, P.R., Clark, F.A., Johnson, D.R., Lovas, F.J., Giguere, P.T., 1974. ApJ 191, 79-82.

Snyder, L.E., Lovas, F.J., Hollis, J.M., Friedel, D.N., Jewell, P.R., Remijan, A., Ilyushin, V.V., Alekseev, E.A., Dyubko, S.F., 2005. ApJ 619, 914-930.

Sil, M., Gorai, P., Das, A., Bhat, B., Etim, E.E., Chakrabarti, S.K., 2017, ApJ (in press)

Taquet, V., Wirström, Eva S., Charnley, S.B., 2016. ApJ 821, 46.

Tercero, B., Kleiner, I., Cernicharo, J., Nguyen, H.V.L., López, A., Muñoz Caro, G.M., 2013. ApJL 770, L13.

Turner, B.E., Apponi, A.J., 2001. ApJ 561, 207-210.

Turner, B.E., 1977. ApJ 213, 75-79.

Tielens, A.G.G.M., 2013. Rev. Mod. Phys. 85, 1021-1081.

Van Dishoeck, E.F., 2004. Annu. Rev. Astron. Astrophys 42, 119-167.

Whittet, D.C.B., 2003. Dust in the Galactic Environment. Institute of Physics Publishing, Bristol.

White, G.J., Araki, M., Greaves, J.S., Ohishi, M., Higginbottom, N.S., 2003. A\&A 407, 589-607.

Ziurys, L.M., Apponi, A.J., Guélin, M., Cernicharo, J., 1995. ApJ 445, L47-L50.

Zuckerman, B., Turner, B.E., Johnson, D.R., Clark, F.O., Lovas, F.J., Fourikis, N., Palmer, P., Morris, M., 1975. ApJ 196, 99-102.

Zuckerman, B., Morris, M., Palmer, P., Turner, B.E., 1972 2264. ApJ 173, $125-129$.

Wakelam, V., Loison, J.-C., Mereau, R., Ruaud, M., 2017. MolAs 6, 22. 